\title{
Sample preparation procedure for the determination of polycyclic aromatic hydrocarbons in petroleum vacuum residue and bitumen
}

\author{
Ewelina Gilgenast • Grzegorz Boczkaj • \\ Andrzej Przyjazny • Marian Kamiński
}

Received: 11 April 2011 /Revised: 20 May 2011 / Accepted: 20 May 2011 /Published online: 7 June 2011

(C) The Author(s) 2011. This article is published with open access at Springerlink.com

\begin{abstract}
This paper describes a novel method of sample preparation for the determination of trace concentrations of polycyclic aromatic hydrocarbons (PAHs) in high-boiling petroleum products. Limits of quantitation of the investigated PAHs in materials of this type range from tens of nanograms per kilogram to $<20 \mu \mathrm{g} / \mathrm{kg}$. The studies revealed that in order to separate most of interferences from the analytes without a significant loss of PAHs, it is necessary to use size exclusion chromatography as the first step of sample preparation, followed by adsorption using normalphase liquid chromatography. The use of orthogonal separation procedure described in the paper allows the isolation of only a group of unsubstituted and substituted aromatic hydrocarbons with a specific range of molar mass. The lower the required limit of quantitation of PAHs, the larger is the scale of preparative liquid chromatography in both steps of sample preparation needed. The use of internal standard allows quantitative results to be corrected for the degree of recovery of PAHs during the sample preparation step. Final determination can be carried out using HPLC-FLD, GC-MS, or HPLC-UV-VIS/DAD. The last technique provides a degree of identification through the acquired UV-VIS spectra.
\end{abstract}

E. Gilgenast · G. Boczkaj · M. Kamiński $(\bowtie)$

Chemical Faculty, Department of Chemical and Process

Engineering, Gdansk University of Technology,

Narutowicza St. 11/12,

80-233 Gdansk, Poland

e-mail: mknkj@chem.pg.gda.pl

\section{A. Przyjazny}

Chemistry \& Biochemistry Department, Kettering University, 1700 West Third Avenue,

Flint, MI 48504, USA
Keywords Sample preparation techniques . Multidimensional liquid chromatography. Group separation - Size exclusion chromatography Normal-phase adsorption chromatography High-boiling petroleum products $\cdot$ Polycyclic aromatic hydrocarbons (PAHs) . Trace analysis

\section{Introduction}

Sample preparation for the determination of trace concentrations of analytes in complex matrices usually includes two steps: extraction of an analyte or analytes from the matrix, followed by their purification [1-3].

This paper describes a novel procedure for the determination of PAHs in such matrices as high-boiling petroleum fractions - so-called vacuum residue products - especially bitumen. Polycyclic aromatic hydrocarbons (PAHs) can be formed in processes of production/distillation of highboiling petroleum products as a result of thermal cracking. The determination of PAHs in petroleum products is an important problem due to their carcinogenic and teratogenic nature and their ubiquitous presence in the environment as a result of emission of dusts, diesel exhausts, and other pollution. The gravity of the problem is signified by recent laws limiting the maximum allowed concentrations of PAHs. For example, in 2006, NIOSH adopted a legal limit of $10 \mu \mathrm{g} / \mathrm{kg}$ for PAH content and $1 \mu \mathrm{g} / \mathrm{kg}$ for benzo[a]pyrene content in technical products.

A major problem in the determination of low or trace content of PAHs in the products mentioned above is the complexity of matrix containing many polycyclic aromatic 
hydrocarbons substituted with aliphatic or alicyclic groups (polycyclic aromatics, PCA).

A standard procedure for the isolation and determination of content of the PCA-containing fraction, including PAHs, was described in the Institute of Petroleum standard IP346, which is widely used all over the world [4]. The procedure is based on a gravimetric determination of the fraction soluble in dimethyl sulfoxide at room temperature. However, the procedure is not sufficiently selective to be used for an effective preparation of a sample of petroleum products for the analysis of trace amounts of PAHs because the extract prepared contains too much aromatic hydrocarbons.

Analytical literature describes the use of size exclusion chromatography (SEC) for sample preparation. The approach involves the isolation of a fraction with a specific range of molar masses. SEC has found numerous uses for sample preparation in the determination of many low-molecular-weight contaminants such as pesticides, drug residues, and PAHs in food, petroleum products, the environment, and other matrices [5-7]. Nerín and Domeño [8] used gel permeation chromatography during the sample preparation step in the determination of PAH content in industrial waste oils. The isolated fraction still contained lipids with low molar masses.

Solid phase extraction is commonly used for sample preparation in the determination of PAHs [9-15], especially in drinking water [9-11]. The technique has also found application in the determination of PAH content in hightemperature tar, as described in Polish standard PN-C82056 [16]. A glass column packed with activated alumina and silica gel and benzene was used to elute the fraction containing PAHs. Alternatively, the procedure calls for the use of Florisil or Sephadex LH-20 as the stationary phase and benzene or methanol, respectively, as the eluting solvents. Nerín and Domeño [8] used a glass column packed with alumina and a mixture of hexane and dichloromethane $(95: 5, v / v)$ as the eluent to purify the fraction obtained through size exclusion. However, the methods making use of adsorption in classical, lowefficiency glass columns are tedious, time-consuming, inaccurate, and imprecise. They consume large volumes of toxic solvents and require repacking columns with a freshly activated sorbent.

Sample preparation procedures based on solid phase extraction (SPE) and used to isolate PAHs from petroleum are also described in technical literature. They make use of Florisil as the stationary phase and hexane as the eluent [17]. Solid phase extraction has a number of advantages, including reduction in volume of solvents used, simplicity of operation, and the possibility of automation (online coupling with high-performance liquid chromatography
(HPLC) or gas chromatography (GC)). At the same time, however, when used for samples having a complex matrix, problems resulting from low recovery of analytes and low reproducibility of results due to nonselective and incomplete desorption can arise.

Our studies revealed that the use of PAH determination procedures described in standards and the literature is completely ineffective for the analysis of PAHs in high-boiling petroleum products. Initially, attempts were undertaken to isolate the low-molecular-mass fraction by means of size exclusion chromatography [9] and to isolate the aromatic fraction using the procedure described in standard IP-346 [4] or by using SPE [17], but these attempts failed. The chromatograms obtained during the separation of fractions isolated from petroleum products reveal the presence of a large number of chemical compounds of diverse polarity and hydrophobicity. The determination of PAHs or other analytes present in trace amounts in such a complex matrix having moderate or strong interactions with the surface of the stationary phase is impossible. Consequently, the development of a new sample preparation procedure was deemed necessary. Size exclusion chromatography was used in the first step, followed by a group separation by means of normal-phase HPLC. In contrast with the lack of effective sample preparation procedures for the determination of trace amounts of PAHs in complex mixtures, such as fractions from vacuum distillation or technical products, the final determination procedures are well developed, provided that the samples practically do not contain aliphatic and alicyclic derivatives of PAHs. Standard methods [9, 16] and their modifications described in the literature are used.

Low efficiency of SPE columns results in the overlapping of interfering substances with the analytes, especially in the case of very complex matrices. The shortcomings of SPE cartridges and classical glass columns lead to the conclusion that a more powerful technique, such as normal-phase HPLC (NP-HPLC), may be required for a satisfactory group separation of complex mixtures. The NP-HPLC stationary phases most commonly used for the determination of group composition of petroleum products were: silica gel [18], alumina, a mixture of silica gel and alumina [19-21], Florisil [22], as well as bonded phases - silica gel modified with cyanopropyl (CN) [23] or aminopropyl (NH2) [24]. These papers dealt with the group separation and group determination and not with the sample preparation. However, they provide information on planning the use of HPLC columns for sample preparation. Saravanabhavan et al. [18] proposed a sample preparation procedure for the simultaneous determination of unsubstituted and alkyl-substituted PAHs in the 
heavy gas oil fraction (boiling range, $287-481^{\circ} \mathrm{C}$ ). Waxes were precipitated first and the extract was separated into five fractions using normal-phase HPLC with a silica column. Already in the 1980s, Wise et al. [25, 26] proposed the use of normal-phase HPLC columns during the sample preparation step for the determination of trace amounts of PAHs in mussels. In the procedures developed by Wise and Saravanabhavan, the elution range of specific groups of analytes was determined by the volume and kind of the mobile phase flowing through the chromatographic column. Adding an HPLC detector to the chromatographic system used for the sample preparation step, which enables complete control over the group separation process, seems to be a better approach.

In the European standard EN 16143 [27], a two-step sample preparation procedure for the determination of the content of benzo $[a]$ pyrene and seven other PAHs is used. The first cleaning step is done with a deactivated silica gel glass column for the separation of low-polar compounds, i.e., PAHs from high-polar compounds, and the second cleaning step shall be performed on Sephadex LH20 for the isolation of the characteristic $\mathrm{PAH}$ molecular weight fraction. Identification and quantification is done by GCmass spectrometry (GC-MS). The procedure is laborconsuming and the intended working range for this method is $0.1-10 \mathrm{mg} / \mathrm{kg}$.

Currently, the most often used PAH separation and determination techniques are GC-MS [28-30] or gas chromatography with flame ionization detection and reverse-phase high-performance liquid chromatography with fluorimetric detection (RP-HPLC-FLD) or (for identification purposes) with diode array detection (RP-HPLCDAD). Separation of PAHs by HPLC is carried out in the reverse-phase mode.

Quantitative determination of PAHs is often accomplished by the external standard (calibration curve) method $[8,18]$ or by the internal standard method [11, 23]. The latter method is particularly suitable for the analytical procedures requiring multistep sample preparation. The addition of an internal standard to a sample before the sample preparation steps are carried out allows a correction for analyte losses. However, different recoveries of the internal standard and analytes originally present in the matrix can present a problem. For this reason, attention should be paid during the addition of internal standard to ensure proper homogenization of a sample. The selection of an appropriate compound as the internal standard can also be a problem. In this paper, the calibration curve method was used for quantitation. The final determination of PAHs in high-boiling petroleum products using internal standard will be discussed in a separate paper.

\section{Experimental}

Materials

\section{Solvents and eluents}

n-Hexane for HPLC (Merck, Darmstadt, Germany), dichloromethane (POCh, Gliwice Poland), acetonitrile HPLC gradient grade (Merck), and deionized water obtained from a Milli $Q$ water purification system (Millipore, Bedford, MA, USA) were used.

\section{Standard solutions}

Benzene (Merck); coronene (Merck); calibration mixture containing naphthalene, acenaphthylene, acenaphthene, fluorene, phenanthrene, anthracene, fluoranthene, pyrene, benzo $[a]$ anthracene, chrysene, benzo[b]fluoranthene, benzo $[k]$ fluoranthene, benzo $[a]$ pyrene, dibenzo $[a, h]$ anthracene, indeno [1,2,3-cd]pyrene, benzo[ghi]perylene at $2,000 \mu \mathrm{g} / \mathrm{mL}$ each component in dichloromethane (Supelco, Bellefonte, PA, USA), and additionally benzo[e]pyrene (Sigma Aldrich, St. Louis, MO, USA), benzo[j]fluoranthene (Sigma Aldrich), and samples of asphalts manufactured at the Gdansk Refinery (Group LOTOS S.A., Poland) at a concentration of $0.05 \mathrm{~g} / \mathrm{mL}$ in dichloromethane were used for analysis.

\section{Chromatographic and SPE columns}

Preparative columns were $250 \times 25 \mathrm{~mm}$ LiChrogel PS-MIX, $10 \mu \mathrm{m}$ (Merck), and 200×16.8 mm Lichrosorb Si60, $10 \mu \mathrm{m}$ (Merck). Analytical columns used were $250 \times 4.6 \mathrm{~mm}$ Spherisorb PAH, $5 \mu \mathrm{m}$, and SPE column packed with Florisil1,000 mg (Macherey-Nagel, Düren, Germany).

\section{Apparatus}

A LaChrom gradient liquid chromatograph (Merck-Hitachi, Darmstadt, Germany) equipped with a four-channel lowpressure gradient system, a L-6200 pump, a Rheodyne Rh$7725 \mathrm{i}$ injector with a $100-\mu \mathrm{L}$ sample loop, a chromatographic column, a thermostat, a model 7450A UV-DAD detector, a model F 1050 fluorimetric detector, HSM software, and a V 7226 six-port two-channel backflush valve (Knauer, Berlin, Germany) were used.

A Merck-Hitachi gradient liquid chromatograph equipped with a four-channel low-pressure gradient system, a L-6200 pump, a Rheodyne Rh-7161 injector with a 1-mL sample loop, a chromatographic column, a thermostat, a model L-3000 UV-DAD detector, a model 1 detector, HSM software, and a V 7226 six-port two-channel backflush valve (Knauer, Berlin, Germany) were also used. 
A Soxtec HT 1043 extraction unit was from Tecator, Höganäs, Sweden.

A solvent evaporation unit consisting of a nitrogen tank, a J.T. Baker polyamide cover equipped with needles and gas connectors, and a metal stand in which flasks with extracts are placed were used.

\section{Procedure}

\section{Isolation of low-molar-mass range fraction}

A PS-MIX $\left(250 \times 25 \mathrm{~mm}, d_{\mathrm{p}}=10 \mu \mathrm{m}\right)$ preparative column, dichloromethane as the mobile phase, and refractive index and UV-DAD detectors connected in series were used in this step. The elution limits of PAHs were determined using the retention times of coronene and benzene as standards. Solutions of petroleum products in dichloromethane were mixed in a 1:1 ratio by volume with a standard solution of coronene and benzene at $0.025 \mathrm{~g} / \mathrm{mL}$ in dichloromethane. Size exclusion separations of solutions of high-boiling petroleum products were carried out both with and without benzene and coronene standards. All samples were separated at $20^{\circ} \mathrm{C}$. The mobile phase flow rate was $4.5 \mathrm{~mL} / \mathrm{min}$ and the injection volume $550 \mu \mathrm{L}$. A low-molar-mass fraction containing PAHs and eluting between benzene and coronene was collected.

\section{Isolation of PAH fraction by NP-HPLC}

The fraction obtained from SEC was evaporated to dryness in a stream of nitrogen. The dry residue was dissolved in $550 \mu \mathrm{L}$ of $n$-hexane and separated by normal-phase HPLC. A silica gel preparative column and UV-DAD (at $\lambda=254 \mathrm{~nm}$ ) and refractive index detectors connected in series were used. The elution limits of PAHs were determined on the basis of the retention times of benzene and coronene. The fraction eluting between 6.5 and $8.4 \mathrm{~min}$ (the start of elution of coronene and the end of benzene elution) was collected. The separation was carried out at $20^{\circ} \mathrm{C}$ using $n$-hexane as the mobile phase at $4.5 \mathrm{~mL} / \mathrm{min}$ and an injection volume of $500 \mu \mathrm{L}$. The backflush valve was switched after $10 \mathrm{~min}$, thus ensuring elution of all polycyclic aromatic hydrocarbons from the column before the backflush was initiated as well as removal of all highly polar components strongly adsorbed on the stationary phase as a single peak following backflush.

\section{Isolation of PAH by SPE}

The fraction obtained from SEC was evaporated to dryness in a stream of nitrogen. The dry residue was dissolved in $550 \mu \mathrm{L}$ of $n$-hexane and separated by SPE.
This step was performed according to the procedure outlined in the Macherey-Nagel application note "PAH from crude oil" [31]. SPE columns packed with Florisil were used in the investigations. Prior to use, the columns were conditioned with $20 \mathrm{~mL}$ of methanol, dried, reconditioned with $20 \mathrm{~mL}$ of $n$-hexane, and dried again. Of the fraction, $180 \mu \mathrm{L}$ was adsorbed on $1 \mathrm{~g}$ of Florisil. The analytes were desorbed from the sorbent bed with $25 \mathrm{~mL}$ of $n$-hexane. The fraction obtained in this manner was chromatographed following solvent exchange and enrichment (see "Separation, identification, and determination of PAH content by RP-HPLC").

\section{Separation, identification, and determination of $P A H$ content by RP-HPLC}

The PAH-rich fraction obtained by the procedure described in "Isolation of low-molar-mass range fraction" and "Isolation of PAH by SPE" was evaporated to dryness in a stream of nitrogen. The dry residue was redissolved in $70 \mu \mathrm{L}$ of acetonitrile. Two detectors connected in seriesUV-DAD (from 220 to $450 \mathrm{~nm}$ ) and fluorimetric-were used in the investigations. Excitation wavelength, $\lambda_{\mathrm{ex}}$, was $275 \mathrm{~nm}$, while the emission wavelength, $\lambda_{\text {em }}$, was $375 \mathrm{~nm}$ for naphthalene, acenaphthene, fluorene, and phenanthrene, and $410 \mathrm{~nm}$ for the remaining PAHs. The Spherisorb PAH column $(5 \mu \mathrm{m}, 250 \times 4.6 \mathrm{~mm})$ was used. The following gradient program was employed: A (acetonitrile), $\mathrm{B}$ (water); $80 \% \mathrm{~A}$ at $40 \mathrm{~min}$ and step to $100 \% \mathrm{~A}$ at $45 \mathrm{~min}$. The separations were carried out at $20^{\circ} \mathrm{C}$ using the mobile phase flow rate of $1 \mathrm{~mL} / \mathrm{min}$ and an injection volume of $50 \mu \mathrm{L}$.

PAH identification was based on:

- Comparison of the retention times of peaks in the chromatograms of samples with those of standards

- Comparison of spectra from 220 to $450 \mathrm{~nm}$, including wavelengths of maxima of individual peaks, with those of PAH standards

The determination of PAH content was carried out by the calibration curve (external standard) method based on the signals of UV-DAD and fluorimetric detectors. The following wavelength program was used for the calibration based on the UV-DAD detector: $270 \mathrm{~nm}$ for naphthalene; $315 \mathrm{~nm}$ for acenaphthylene; $265 \mathrm{~nm}$ for acenaphthene and fluorene; $254 \mathrm{~nm}$ for phenanthrene, anthracene, and fluoranthene; $270 \mathrm{~nm}$ for pyrene; $285 \mathrm{~nm}$ for benzo[ $a]$ anthracene and chrysene; $287 \mathrm{~nm}$ for benzo[b]fluoranthene; $293 \mathrm{~nm}$ for benzo[ $k]$ fluoranthene and benzo $[a]$ pyrene; and $290 \mathrm{~nm}$ for dibenzo $[a, h]$ anthracene, indeno[1,2,3-cd]pyrene, benzo $[g h i]$ perylene, benzo $[e]$ pyrene, and benzo[j]fluoranthene. Five-point calibration curves were prepared for individual PAHs at concentrations ranging from 50 to $1,000 \mathrm{ng} / \mathrm{mL}$ for 
the UV-DAD detector and 0.5 to $32.0 \mathrm{ng} / \mathrm{mL}$ for the fluorimetric detector. During the analysis of real samples, the HPLC column was backflushed right after the elution of PAHs.

\section{Determination of $P A H$ recovery from the investigated samples}

To solutions of road asphalt 50/70 having concentrations of $0.05 \mathrm{~g} / \mathrm{mL}$ in dichloromethane, $10 \mu \mathrm{L}$ of PAH standards at $200 \mu \mathrm{g} / \mathrm{mL}$ in dichloromethane was added. Next, the samples were prepared according to the procedure described in "Isolation of low-molar-mass range fraction," "Isolation of PAH fraction by NP-HPLC," and "Isolation of PAH by SPE."The recovery was determined from the following relationship:

$R=\Delta C_{i(\mathrm{cal})} / \Delta C_{i(\text { true })} \times 100 \%$

where $\Delta C_{i}$ (cal) is the concentration of the $i$ th analyte determined from the calibration curve and $\Delta C_{i}$ (true) is the true concentration of the $i$ th analyte in the analyzed solution.

The limit of quantitation was taken as the concentration corresponding to the signal five times as great as the noise corrected for the recovery.

Each experiment was repeated at least twice. The results presented in the paper are average values.

\section{Results and discussion}

Preparative size exclusion chromatography (P-GPC/P-SEC) as the first step in sample preparation for the analysis of trace amounts of PAHs in petroleum products

The first stage of sample preparation involved the selective isolation of the fraction containing PAHs and differing in molar mass from the majority of other highboiling components of petroleum products by means of size exclusion chromatography. This stage enabled the removal of components having higher molar masses than PAHs and eluted earlier than PAHs. The elution range of PAHs was determined using benzene and coronene as standards. Coronene has a molar mass slightly higher $\left(\mathrm{C}_{24} \mathrm{H}_{12}, M=300 \mathrm{Da}\right)$ than that of PAHs (dibenzo[ $\left.a, h\right]$ anthracene $\mathrm{C}_{22} \mathrm{H}_{14}$ has the highest molar mass of all PAHs, equal to $278 \mathrm{Da}$ ). To mark the start of fraction collection, dibenzo $[a, h]$ anthracene can also be used in place of coronene. Dibenzo $[a, h]$ anthracene has a larger molecular diameter but slightly lower molar mass than coronene. As a result of small differences in those two properties, both compounds are eluted at the same time in size exclusion chromatography. On the other hand, benzene $\left(\mathrm{C}_{6} \mathrm{H}_{6}, M=78 \mathrm{Da}\right)$ determines the lower limit of elution, its molar mass being lower than that of naphthalene $\left(\mathrm{C}_{10} \mathrm{H}_{28}, M=128 \mathrm{Da}\right)$. To lower the limit of quantitation for $\mathrm{PAHs}$, the scale of separation process was increased using a preparative SEC column $\left(d_{\mathrm{c}}=\right.$ $\left.25 \mathrm{~mm}, L_{\mathrm{c}}=250 \mathrm{~mm}, d_{\mathrm{p}}=10 \mu \mathrm{m}\right)$. The use of preparative chromatography enabled isolation in one step substantially larger amounts of the fraction rich in PAHs than an analytical column would allow. Examples of SEC chromatograms of a sample of petroleum products with (Fig. 1a) and without (Fig. 1b) benzene and coronene standards are shown in Fig. 1.

An inspection of Fig. 1 reveals that during this step of sample preparation, the isolated fraction rich in PAHs was stripped of the majority of high-boiling components of petroleum products. The components isolated at this stage include PAHs and other hydrocarbons and organic compounds with various substituents having molar masses close to those of PAHs.

A styrene-divinylbenzene copolymer with a specific range of pore sizes, from 50 to $50,000 \AA$, was used as the stationary phase during the first stage of the sample preparation.

Due to a relatively high cost of a preparative column packed with styrene-divinylbenzene, an attempt was made to replace it with a traditional normal- or reverse-phase packing. To eliminate sorptive interactions and ensure size exclusion mechanism, mobile phases with very high elution strengths were used for each of the columns tested. Polar stationary phases included $\mathrm{CN}$, DIOL, and $\mathrm{NH}_{2}$ and were tested with polar mobile phases such as dichloromethane/ methanol $1: 1$ or tetrahydrofuran/methanol $7: 3$, while the non-polar stationary phase $\mathrm{C} 18$ was studied with a nonpolar mobile phase, such as $n$-hexane. Analytical columns were used in the investigations. The studies revealed that despite using mobile phases with a high eluent strength, it was impossible to eliminate sorptive interactions between normal-phase packings and polar, low-molar-mass components of the examined samples and, similarly, between reverse-phase stationary phases and non-polar components of the investigated samples. In both cases (normal and reversed phases), a large fraction of higher-molecular-mass components had retention times similar to those of benzene and coronene. This indicates a mixed separation mechanism in spite of using eluents with high elution strengths. Consequently, the use of a styrene-divinylbenzene copolymer as the SEC stationary phase was deemed necessary.

Normal-phase adsorption as the second step of sample preparation for the determination of trace PAH amounts in petroleum products

The use of size exclusion chromatography as a sample preparation step for the determination of trace PAH amounts 
Fig. 1 Chromatograms of a sample of road asphalt 50/70 with benzene and coronene standards added (A) and without the standards (B) separated by preparative size exclusion chromatography. Column, $250 \times 25-\mathrm{mm}$ i.d., $d \mathrm{p}=10 \mu \mathrm{m}$ preparative PS-MIX. Eluent: dichloromethane; mobile phase flow rate, $4.5 \mathrm{~mL} / \mathrm{min}$. Injection volume, $550 \mu \mathrm{L}$. Detector: refractive index. Peak designations: 1 components of petroleum, 2 benzene + coronene. The range of collection of the PAH-containing fraction is marked with arrows
A

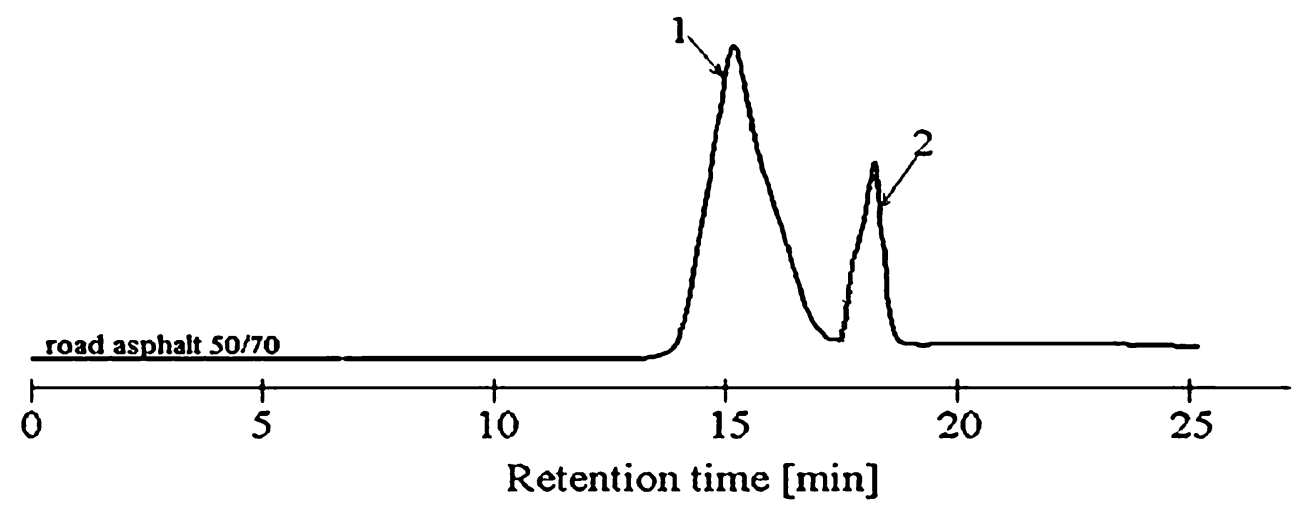

B

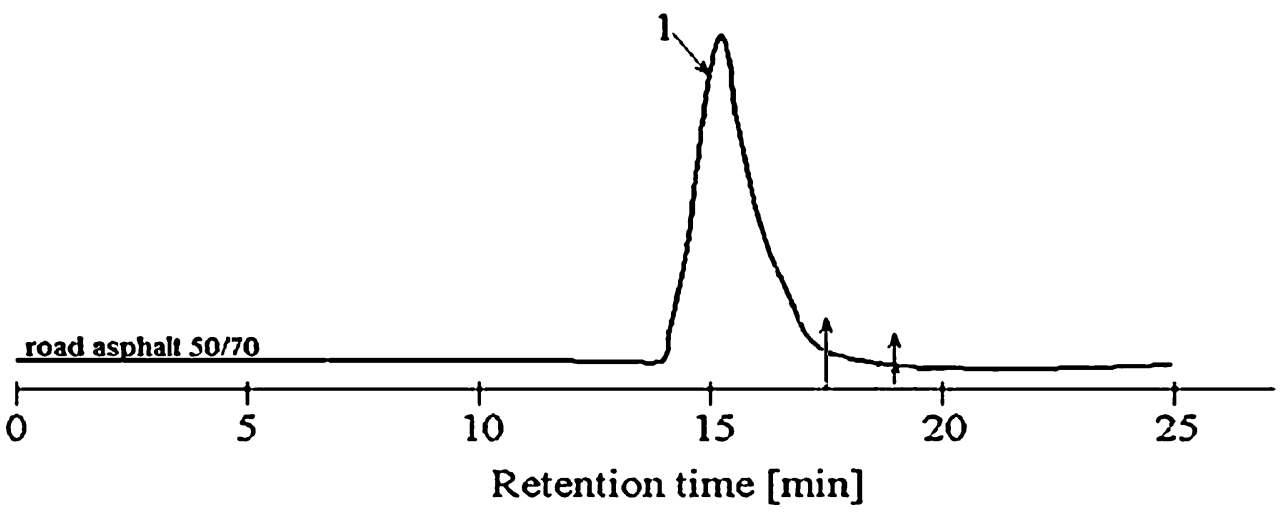

in technical products was found to be insufficient. The isolated fraction contained large amounts of compounds with molar masses similar to those of PAHs and with similar or higher polarity. This precludes final determination by means of RP-HPLC. Adsorption was employed for further purification of the fraction rich in PAHs. SPE in the reverse phase or normal phase modes could conceivably be applied for a group separation with respect to the polarity of functional groups. However, the use of HPLC seems to be a better choice than SPE due to the very complex composition of the fraction isolated by SEC. Although HPLC is more expensive and less amenable to automation, it ensures improved selectivity of separation and considerably better reproducibility of the results. Additionally, by using HPLC detectors (refractive index, fluorescence, or UV), the elution range of the fraction of interest can be accurately determined. The limit of quantitation can be lowered using a semi-preparative or preparative HPLC column.

An example of typical chromatogram of the PAH-rich fraction of petroleum products from the SEC isolation step obtained by normal-phase chromatography is shown in Fig. 2.
As Fig. 2b reveals, the fractions isolated by SEC still contain substantial amounts of polar compounds of low molar masses, which would be strongly retained by the stationary phase, but by using backflush are eluted as a single peak with the retention time twice that of the backflush point. These strongly polar compounds can be removed from the HPLC column using either a step elution with the mobile phase of higher elution strength than $n$-hexane or by backflush. The former solution is time-consuming as a result of column reconditioning, i.e., stationary phase surface equilibration with the solvent of low elution strength. Consequently, using backflush was far more advantageous, the more so because the mobile phase flow rate can be increased during backflush, thus reducing its time. It should be noted, however, that backflush is only possible for very well-packed and stable HPLC columns.

A refractive index, UV-DAD or UV-254, detector can be used during both sample preparation steps. However, the UVDAD detector was used in this study so that the UV spectra of individual sample components could be monitored. 
Fig. 2 Chromatograms obtained in the second step of sample preparation using preparative NP-HPLC for a standard solution containing benzene and coronene (A) and for the PAH-rich fraction of road asphalt 50/70 obtained from size exclusion chromatography $(\mathbf{B})$. Column, $200 \times 16.8 \mathrm{~mm}$, $d \mathrm{p}=10 \mu \mathrm{m}$, preparative packed with silica gel Si60. Eluent: $n$-hexane; mobile phase flow rate, $4.5 \mathrm{~mL} / \mathrm{min}$. Injection volume, $500 \mu \mathrm{L}$. Detector: UV at $254 \mathrm{~nm}$. Peak designations: 1 benzene, 2 coronene, 3 - components of road asphalt $50 / 70$ with lower polarity, 4 components of road asphalt $50 / 70$ with higher polarity. $B F$ backflush point. The range of fraction collection is marked with arrows

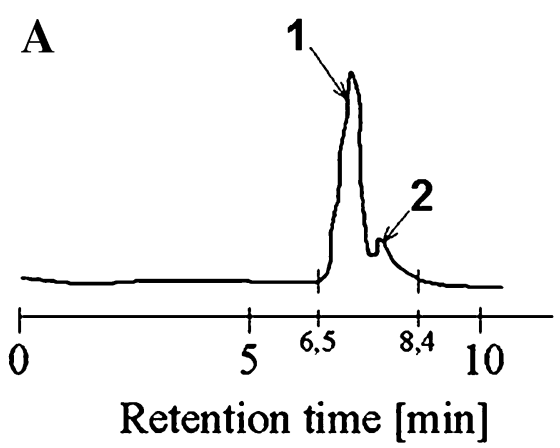

B

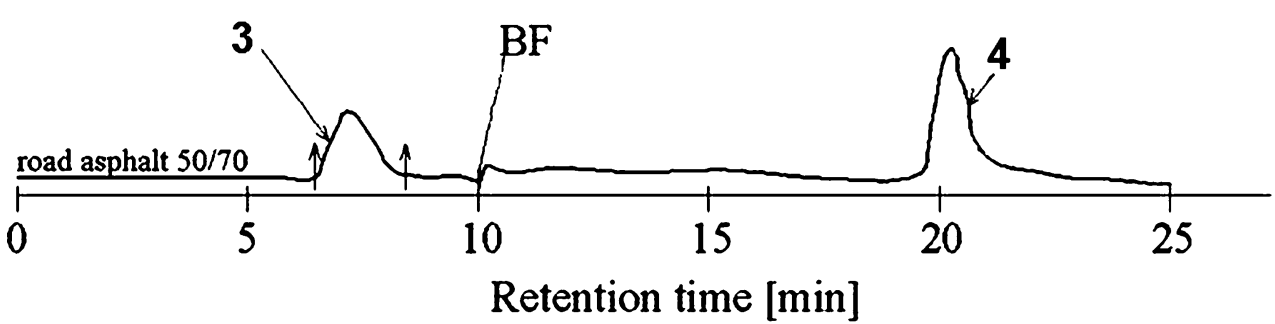

The recoveries of PAHs using the two-step procedure described in this paper-SEC in the first step followed by normal-phase adsorption accomplished with NP-HPLC or SPE-are listed in Table 1.
An inspection of the data in Table 1 reveals that the combination of SEC in the first step and NP-HPLC during the second step provides satisfactory PAH recoveries, exceeding $80 \%$ (except for volatile components, i.e.,
Table 1 Analyte recovery values $(R)$ for PAHs

\begin{tabular}{|c|c|c|c|c|}
\hline \multirow[t]{3}{*}{ Analyte } & \multicolumn{4}{|c|}{ Techniques used during sample preparation } \\
\hline & \multicolumn{2}{|c|}{ 1st step/2nd step (GPC/NP-HPLC) } & \multicolumn{2}{|c|}{ 1st step/2nd step (GPC/NP-SPE) } \\
\hline & $R, n=3(\%)$ & RSD (\%) & $\mathrm{R}, n=3(\%)$ & RSD (\%) \\
\hline Naphthalene & 5.9 & 2.3 & 4.6 & 1.6 \\
\hline Acenaphthene & 7.8 & 3.0 & 7.0 & 3.8 \\
\hline Acenaphthylene & 8.9 & 2.6 & 7.6 & 3.2 \\
\hline Fluorene & 58.0 & 2.0 & 11.0 & 5.0 \\
\hline Phenanthrene 85.0 & 3.6 & 12.0 & 7.3 & \\
\hline Anthracene & 96.0 & 3.3 & 15.0 & 4.0 \\
\hline Fluoranthene 87.0 & 2.8 & 22.0 & 4.2 & \\
\hline Pyrene & 94.0 & 3.1 & 46.6 & 9.6 \\
\hline Benzo $[a]$ anthracene & 100.0 & 5.0 & 42.0 & 7.1 \\
\hline Chrysene 100.0 & 3.9 & 38.0 & 4.3 & \\
\hline Benzo[j]fluoranthene & 92.0 & 2.5 & 52.0 & 7.0 \\
\hline Benzo[e]pyrene & 98.0 & 2.7 & 37.0 & 9.4 \\
\hline Benzo $[b]$ fluoranthene & 100.0 & 3.4 & 56.0 & 4.1 \\
\hline Benzo $[k]$ fluoranthene & 94.0 & 2.8 & 57.0 & 7.1 \\
\hline Benzo $[a]$ pyrene & 100.0 & 4.1 & 66.0 & 4.7 \\
\hline Dibenzo $[a, h]$ anthracene 100.0 & 3.9 & 46.0 & 10.2 & \\
\hline Indeno[1,2,3-cd]pyrene & 91.0 & 3.0 & 48.0 & 9.7 \\
\hline Benzo[ghi]perylene & 100.0 & 3.5 & 39.0 & 5.1 \\
\hline
\end{tabular}


naphthalene, acenaphthene, acenaphthylene, and fluorene). The use of NP-HPLC instead of SPE in the second step results in significantly higher recoveries due to the higher column efficiency of the HPLC columns compared with the SPE columns. The low efficiency of SPE columns leads to overlapping of the analyte fraction with the fraction of more polar components. The limitation of the presented procedure is its inability to determine volatile hydrocarbons - naphthalene, acenaphthene, and acenaphthylene. This shortcoming results from the need for solvent exchange after each sample preparation step. A schematic diagram of the two-step sample preparation procedure for the determination of trace PAH content in technical products described in this paper is depicted in Fig. 3.

Final determination of PAHs by RP-HPLC

The final determination of PAHs was carried out using a modified procedure described in Polish Standard PN-EN ISO 17993 [9]. The modification involved an extension of the standard procedure to include two additional hydrocarbons, benzo[j]fluoranthene and benzo[e]pyrene, whose maximum allowed concentrations are also regulated. An
Fig. 3 Flowchart for the procedure of sample preparation for the determination of PAHs in technical products proposed in the present work

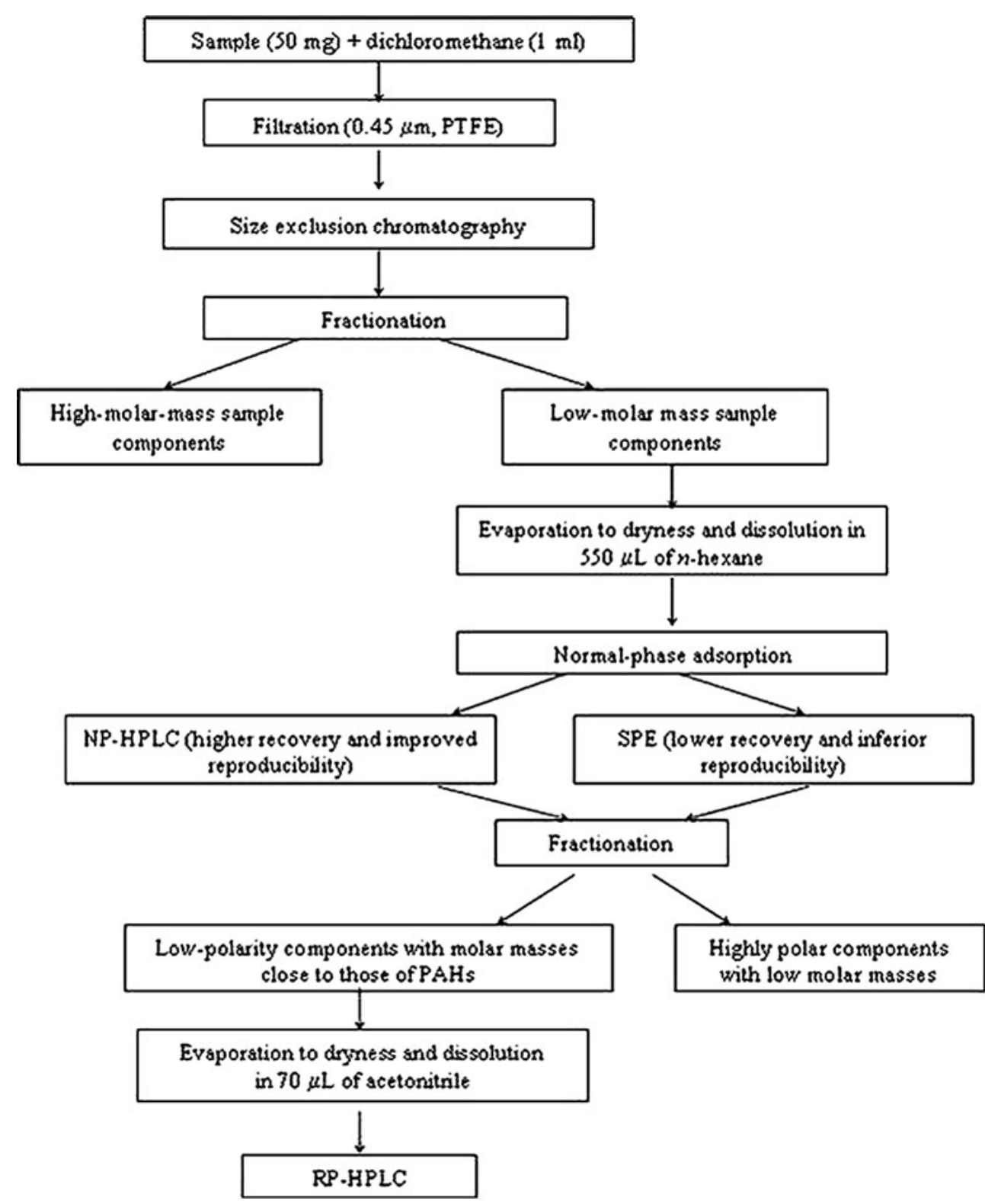


additional argument for using step elution was the attempt to maximize reproducibility of retention times, which are identification parameters when using a fluorescence detector. The reproducibility of retention times is better in step elution than in gradient elution due to the imperfect synchronization of cyclic operation of proportioning valves in HPLC chromatographs utilizing the so-called lowpressure gradient. The type of stationary phase, composition of the mobile phase, and the elution program were all optimized. The optimum conditions were found to be Spherisorb PAH as the stationary phase and step elution using a mixture of acetonitrile and water as the mobile phase. Limits of quantitation obtained by the developed procedure for the fluorescence and UV-DAD detectors are compiled in Table 2.

Examples of the chromatograms of 18 standards obtained using UV-DAD detection with wavelength programming and fluorimetric detection as well as of a chromatogram of the PAH-rich fraction isolated from a sample of road asphalt 50/70 according to the procedure discussed in this paper obtained using UV-DAD and fluorimetric detection are shown in Fig. 4.

Chrysene and benzo $[a]$ pyrene were identified and determined in the sample of road asphalt 50/70. The identified PAHs occurred in real samples at concentrations of 32.8 and 1,184.5 ppb, respectively. Unsubstituted PAHs and those substituted with aliphatic and alicyclic groups occur originally in petroleum $[18,32]$. As a result of the range of distillation temperatures, the unsubstituted PAHs studied in this paper largely do not occur in the vacuum residue. The presence of these analytes in bitumen is probably caused by thermal cracking. The reported concentrations are approximate since the present paper aimed at determining the recovery and limits of quantitation of PAHs. To consider the reported concentrations as accurate would require validation of the developed procedure using a certified reference material. These problems will be discussed in a later paper.

\section{Conclusions}

On the basis of the results obtained, the following conclusions can be drawn:

- The paper describes the procedure for the sample preparation of petroleum products for the determination of trace amounts of PAHs.

- The use of size exclusion chromatography with a preparative column as the first step of sample preparation is necessary, but not sufficient. The use of normal-phase adsorption in the second step enables the determination of very small amounts of PAHs in the investigated matrices with a high and reproducible recovery.

- The only stationary phase suitable for the first sample preparation step is the styrene-divinylbenzene copolymer.

The separate use of size exclusion chromatography and adsorption chromatography during the sample
Table 2 Limit of quantitation for PAHs analytes in highboiling petroleum products

\begin{tabular}{lcc}
\hline Analyte & RP-HPLC-UV-DAD $(\mu \mathrm{g} / \mathrm{kg})$ & RP-HPLC-FLD $(\mu \mathrm{g} / \mathrm{kg})$ \\
\hline Naphthalene & 339.01 & 42.45 \\
Acenaphthene & 256.42 & - \\
Acenaphthylene & 224.75 & 20.15 \\
Fluorene & 18.82 & 2.12 \\
Phenanthrene & 4.17 & 0.09 \\
Anthracene & 2.08 & 0.22 \\
Fluoranthene & 21.84 & 0.20 \\
Pyrene & 13.83 & 0.05 \\
Benzo[a]anthracene & 8.00 & 0.23 \\
Chrysene & 6.00 & 0.04 \\
Benzo $[j]$ fluoranthene & 38.04 & 7.05 \\
Benzo $[e]$ pyrene & 53.06 & 1.03 \\
Benzo $[b]$ fluoranthene & 34.31 & 7.31 \\
Benzo $k]$ fluoranthene & 32.98 & 0.27 \\
Benzo[a]pyrene & 33.00 & 0.23 \\
Dibenzo[ $a, h]$ anthracene & 24.75 & 1.32 \\
Indeno[1,2,3-cd]pyrene & 37.36 & 0.89 \\
Benzo $[g h i]$ perylene 51. & 96 & 10.02 \\
\hline
\end{tabular}


Fig. 4 Chromatograms obtained using UV-DAD detection with wavelength programming (A) and fluorimetric detection (B) for the separation of 18 PAH standards $(\mathbf{A}, \mathbf{B})$ and the fraction containing PAHs from road asphalt 50/70 prepared according to the procedure described in this work (C).

Separation and detection conditions are described in "Experimental." Peak designation: 1 naphthalene, 2 acenaphthylene, 3 acenaphthene, 4 fluorene, 5 phenanthrene, 6 anthracene, 7 fluoranthene, 8 pyrene, 9 benzo [a] anthracene, 10 chrysene, 11 benzo $[b]$ fluoranthene, 12 benzo $[k]$ fluoranthene, 13 benzo $[a]$ pyrene, 14 dibenzo $[a, h]$ anthracene, 15 indeno[1,2,3-cd]pyrene, 16 benzo[ghi]perylene, 17 benzo[j] fluoranthene, 18 benzo[e]pyrene, 19 highly polar components of road asphalt 50/70 eluted during backflush. $B F$ backflush point
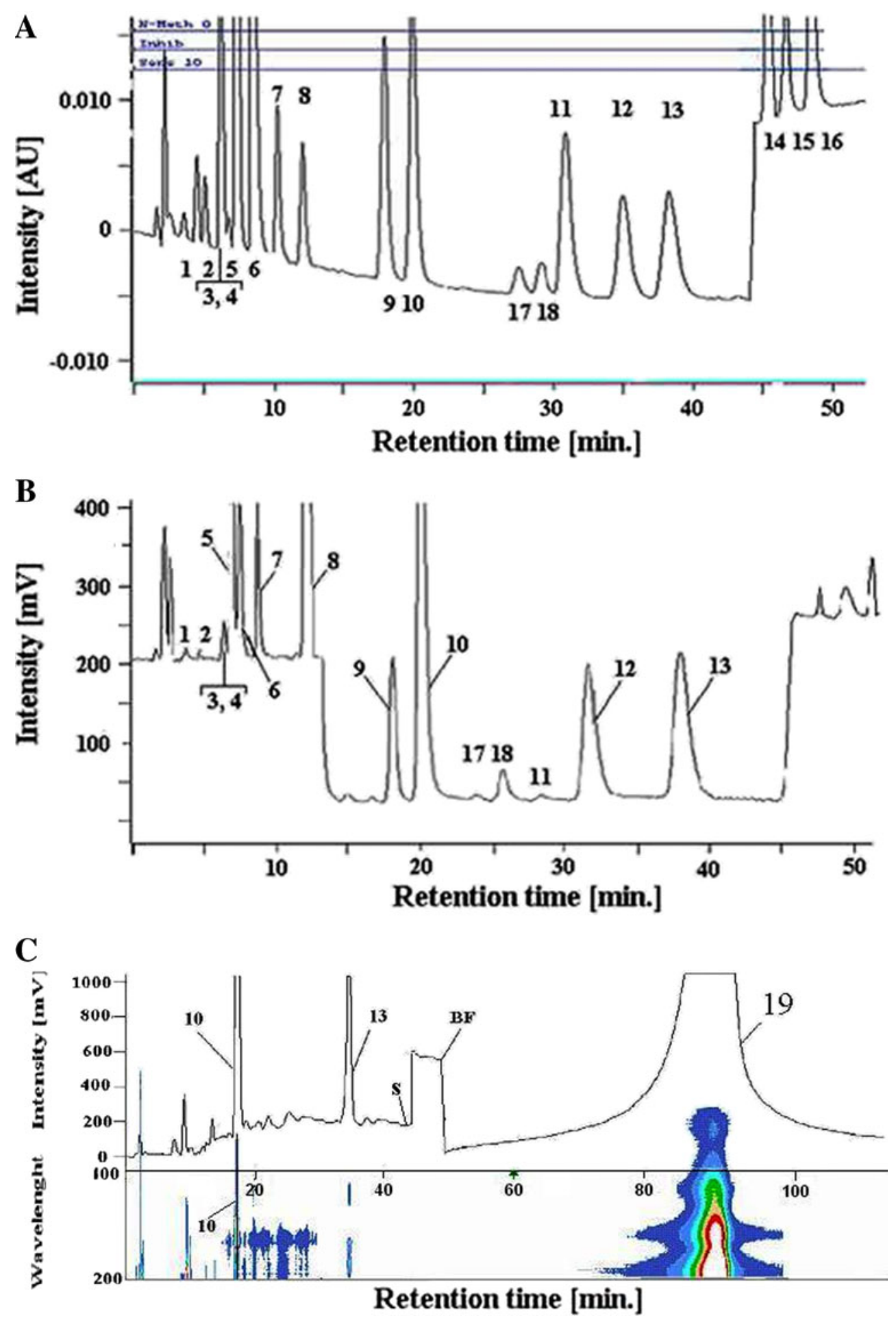

preparation step does not allow selective isolation of PAHs from the hydrocarbon matrix of petroleum products, thus precluding trace analysis of PAHs in these materials.

- Normal-phase HPLC with a preparative column is superior to normal-phase SPE.
- The advantages of HPLC compared with SPE during the second step of sample preparation include the following:

- High efficiency and good reproducibility of efficiency and selectivity of chromatographic columns compared with SPE columns 
- Possibility of monitoring the range of fraction collection using an HPLC detector (refractive index, UV, or fluorescence)

- Possibility of using backflush in the HPLC column to elute highly polar components, which ensures a substantial reduction in time of analysis

- Improved recovery of PAHs and lower relative standard deviation values

- In order to reduce the limit of quantitation for the entire procedure, preparative liquid chromatography is preferred.

- Final determination of PAHs was carried out by RPHPLC with UV-DAD and fluorimetric detection.

Acknowledgments The authors gratefully acknowledge the financial support from the State Committee for Scientific Research, Warsaw, Poland (contract no. N N523 4531 33).

Open Access This article is distributed under the terms of the Creative Commons Attribution Noncommercial License which permits any noncommercial use, distribution, and reproduction in any medium, provided the original author(s) and source are credited.

\section{References}

1. Moret S, Conte LS (2000) J Chromatogr A 882:245-253

2. Purcaro G, Moret S, Conte LS (2009) Meat Sci 81:275-280

3. Tsai JH, Chen SJ, Huang KL, Lin YC, Leec WJ, Lin CC, Lin WY (2010) J Hazard Mater 179:237-243

4. Standard IP 346/92: Determination of polycyclic aromatics in unused lubricating base oils and asphaltene free petroleum fractionsdimethyl sulphoxide extraction refractive index method

5. Furusawa N, Ozaki A, Nakamura M, Morita Y, Okazaki KJ (1999) J Chromatogr A 830:473-476

6. Rimkus GR, Rummler M, Nausch I (1996) J Chromatogr A 737:9-14

7. Venkatesan MI, Northrup T, Phillip ChR (2002) J Chromatogr A 942:223-230

8. Nerin C, Domeno C (1999) Analyst 124:67-70

9. Polish standard PN-EN ISO 17993 "Jakość wody. Oznaczanie 15 wielopierścieniowych węglowodorów aromatycznych (WWA) w wodzie metodą HPLC z detekcją fluorescencyjną po ekstrakcji ciecz-ciecz" [Water quality. Determination of 15 polycyclic aromatic hydrocarbons (PAHs) in water using HPLC with fluorimetric detection following liquid-liquid extraction]

10. Chen Y, Zhu L, Zhou R (2007) J Hazard Mater 141:148-155

11. Pillai I, Ritchie L, Heywood R, Wilson G, Pahlavanpour B, Setford S, Saini SJ (2005) J Chromatogr A 1064:205-212

12. Moret S, Purcaro G, Conte LS (2005) Eu J Lipid Sci Technol 107:488-496

13. Santos JL, Aparicio I, Alonso E (2007) Anal Chim Acta 605:102109

14. Moret S, Conte LS (2002) J Sep Sci 25:96-100

15. Da Porto C, Moret S, Soldera S (2006) Anal Chim Acta 563:396400

16. Polish standard PN-C-82056: 2000, Produkty weglopochodne Oznaczanie zawartości wielopierścieniowych węglowodorów aromatycznych (WWA) w smole koksowniczej metodą cieczowej chromatografii kolumnowej [Coal derived products - determination of the content of polycyclic aromatic hydrocarbons (PAHs) in high-temperature tar by column liquid chromatography]

17. Snyder LR (1961) Anal Chem 33:1527-1532

18. Saravanabhavan G, Helferty A, Hodson PV, Brown RS (2007) J Chromatogr A 1156:124-133

19. Lancas FM, Carniho E, Deane GHN, Camilo MCF (1989) HRC 12:368-395

20. Rashid HA, Fakhn NA, Dekran SB, Abdulla NI (1989) Fuel Sci Technol Int 7:281-289

21. Aceves M, Grimalt J, Albaiges J, Broto F, Comellas L, Gassiot M (1988) J Chromatogr A 436:503-515

22. Baumeister W, Zens B, Viene O, Fresenius Z (1989) Anal Chem 333:710-718

23. Karlesky L, Rollie ME, Warner M (1989) Anal Chem 58:11871193

24. Obuchi A, Aoyama H, Obuchi H (1984) J Chromatogr A 312:247-258

25. Wise SA, Chesler SN, Hertz HS, May WE, Guenther FR, Hilpert LR (1980) Analytical Techniques in Environmental Chemistry 41-51

26. Wise SA, Chesler SN, Hertz HS, Hilpert LR, May WE, Parris RM (1980) Anal Chem 52:1828-1833

27. EN 16143:2010. Petroleum products - determination of content of benzo $(a)$ pyrene $(\mathrm{BaP})$ and selected polycyclic aromatic hydrocarbons (PAH) in extender oils-procedure using double LC cleaning and $\mathrm{GC} / \mathrm{MS}$ analysis

28. Gomes RB, Nogueira R, Oliveira JM, Peixoto J, Brito AG (2009) Environ Sci Pollut Res 16:671-678

29. Purcaro G, Morrison P, Moret S, Conte LS, Marriott PJ (2007) J Chromatogr A 1161:284-291

30. Christensen A, Ostman C, Westerholm R (2005) Anal Bioanal Chem 381:1206-1216

31. SPE Application Guide (2006) Macherey-Nagel, Germany

32. Boduszynski MM (1988) Energy Fuels 2:597-613 